\title{
A COMBINATORIAL PROBLEM ON ABELIAN GROUPS
}

\author{
MARSHALL HALL, JR.
}

1. Introduction. Suppose we are given a finite abelian group $A$ of order $n$, the group operation being addition. If

$$
\left(\begin{array}{c}
a_{1}, a_{2}, \cdots, a_{n} \\
c_{1}, c_{2}, \cdots, c_{n}
\end{array}\right)
$$

is a permutation of the elements of $A$, then the differences $c_{1}-a_{1}$ $=b_{1}, \cdots, c_{n}-a_{n}=b_{n}$ are $n$ elements of $A$, not in general distinct, such that $\sum_{i=1}^{n} b_{i}=\sum_{i=1}^{n} c_{i}-\sum_{i=1}^{n} a_{i}=0$, since the sum of the $c^{\prime} \mathrm{s}$ and the sum of the $a$ 's are each the sum of all the elements of $A$. The problem is to show that conversely given a function $\phi(i)=b_{i}$, $i=1, \cdots, n$, with values $b_{i}$ in $A$ subject only to the condition that $\sum_{i=1}^{n} b_{i}=0$, then there exists a permutation

$$
\left(\begin{array}{l}
a_{1}, \cdots, a_{n} \\
c_{1}, \cdots, c_{n}
\end{array}\right)
$$

of the elements of $A$ such that $c_{i}-a_{i}=b_{i}, i=1, \cdots, n$, if the $b$ 's are appropriately renumbered. This problem ${ }^{1}$ is solved in this paper.

\section{Solution of the problem.}

THEOREM. Given a function $\phi(i)=b_{i}, i=1, \cdots, n$, with $b_{i}$ in $A$, an additive abelian group of order $n$, subject to the condition $\sum_{i=1}^{n} b_{i}=0$, there exists a permutation

$$
\left(\begin{array}{l}
a_{1}, \cdots, a_{n} \\
c_{1}, \cdots, c_{n}
\end{array}\right)
$$

of the elements of $A$ such that $c_{i}-a_{i}=b_{i}, i=1, \cdots, n$, the $b$ 's being appropriately renumbered.

Proof. If we take $a_{1}, a_{2}, \cdots, a_{n}$ as the elements of $A$ in an arbitrary but fixed order, the problem consists in renumbering the $b$ 's so that $a_{1}+b_{1}=c_{1}, a_{2}+b_{2}=c_{2}, \cdots, a_{n}+b_{n}=c_{n}$ are all distinct.

It is sufficient to prove that given a permutation whose differences are $b_{1}, b_{2}, \cdots, b_{n-2}, b_{n-1}^{\prime}, b_{n}^{\prime}$, we can find another whose differences $b_{1}, b_{2}, \cdots, b_{n-2}, b_{n-1}, b_{n}$ are the same except that two of them, $b_{n-1}^{\prime}$ and $b_{n}^{\prime}$, have been replaced by two others, $b_{n-1}$ and $b_{n}$, with the

Presented to the Society, October 27, 1951; received by the editors July 24, 1951.

${ }^{1}$ For the cyclic group this shows the truth of a conjecture of Dr. George Cramer. 
same sum $b_{n-1}+b_{n}=b_{n-1}^{\prime}+b_{n}^{\prime}$. For the identical permutation has differences $0,0, \cdots, 0$ and we may replace these differences two at a time to give differences $b_{1}, w_{2}, 0, \cdots, 0 ; b_{1}, b_{2}, w_{3}, 0, \cdots, 0 ; \cdots$; $b_{1}, b_{2}, \cdots, b_{n-1}, w_{n}$ where $w_{2}=-b_{1}, w_{3}=-b_{1}-b_{2}, \cdots, w_{n}=-b_{1}$ $-b_{2}-\cdots-b_{n-1}=b_{n}$.

Thus we suppose given an incomplete permutation

$$
\left(\begin{array}{l}
a_{1}, \cdots, a_{n-2}, \cdots \\
c_{1}, \cdots, c_{n-2}, \cdots
\end{array}\right)
$$

with differences $b_{1}, b_{2}, \cdots, b_{n-2}$ which we represent by a table:

$$
\begin{aligned}
& a_{1} a_{2} \cdots a_{n-2} a_{n-1} a_{n} \\
& b_{1} b_{2} \cdots b_{n-2} \quad b_{n-1} b_{n} \\
& c_{1} c_{2} \cdots c_{n-2} \quad u_{-1} u_{0} \text {. }
\end{aligned}
$$

In this table $a_{i}+b_{i}=c_{i}, i=1, \cdots, n-2$, and we have left over two $a$ 's, two $b$ 's, and the two elements $u_{0}$ and $u_{-1}$ which together with $c_{1}, c_{2}, \cdots, c_{n-2}$ make up all the elements of $A$. Here we have

$$
\sum_{i=1}^{n-2} a_{i}+a_{n-1}+a_{n}+\sum_{i=1}^{n-2} b_{i}+b_{n-1}+b_{n}=\sum_{i=1}^{n-2} c_{i}+u_{-1}+u_{0}
$$

since each of $\sum_{i=1}^{n} a_{i}$ and $\sum_{i=1}^{n-2} c_{i}+u_{-1}+u_{0}$ is the sum of all the elements of $A$ and by hypothesis $\sum_{i=1}^{n} b_{i}=0$. But since $a_{i}+b_{i}=c_{i}$, $i=1, \cdots, n-2$, we shall have from $(2.2)$

$$
a_{n-1}+a_{n}+b_{n-1}+b_{n}=u_{-1}+u_{0} \text {. }
$$

In (2.3) if one $a$ plus one $b$ is one of the $u$ 's, then the other $a$ plus the other $b$ is the remaining $u$ and we can complete (2.1) to a full permutation with differences $b_{1}, \cdots, b_{n}$ as was to be done. If not, then the equation $x+b_{n-1}=u_{-1}$ has as its solution $x=a_{r_{1}}, 1 \leqq r_{1} \leqq n-2$. Now in (2.1) let us replace $b_{r_{1}}$ and $c_{r_{1}}$ by $b_{n-1}$ and $u_{-1}$ leading to the following table:

$$
\begin{array}{ll}
a_{1} \cdots a_{r_{1}} \cdots a_{n-2} a_{n-1} a_{n} & \\
b_{1} \cdots b_{n-1} \cdots b_{n-2} & b_{r_{1}} b_{n} \\
c_{1} \cdots u_{-1} \cdots c_{n-2} & u_{0} c_{r_{1}}
\end{array}
$$

and as from (2.1) we have

$$
a_{n-1}+a_{n}+b_{r_{1}}+b_{n}=u_{0}+c_{r_{1}} \text {. }
$$

In (2.5) if one $a$ plus one $b$ is $u_{0}$ or $c_{r_{1}}$, the same holds for the other $a, b$, and $c_{r_{1}}$ or $u_{0}$ and we have found a solution to the problem. If 
not, the equation $x+b_{r_{1}}=u_{0}$ has a solution $x=a_{r_{2}}$ with $1 \leqq r_{2} \leqq n-2$. Let us then replace $b_{r_{2}}$ and $c_{r_{2}}$ by $b_{r_{1}}$ and $u_{0}$ in (2.4) leading to another incomplete permutation. If we continue this process for $i$ steps, we have (if $a_{r_{1}}, \cdots, a_{r_{i}}$ are all different)

$$
\begin{aligned}
& a_{1} \cdots a_{r_{1}} a_{r_{2}} a_{r_{3}} \cdots a_{r_{i}} \quad \cdots a_{n-2} a_{n-1} a_{n} \\
& b_{1} \cdots b_{n-1} b_{r_{1}} b_{r_{2}} \cdots b_{r_{i-1}} \cdots b_{n-2} \quad b_{r_{i}} b_{n} \\
& c_{1} \cdots u_{-1} \quad u_{0} c_{r_{1}} \cdots c_{r_{i-2}} \cdots c_{n-2} \quad c_{r_{i-1}} c_{r_{i}} \text {. }
\end{aligned}
$$

At the $i$ th stage we solve the equation $x+b_{r_{i}}=c_{r_{i-1}}$. If this $x$ is $a_{n-1}$ or $a_{n}$, the relation

$$
a_{n-1}+a_{n}+b_{r_{i}}+b_{n}=c_{r_{i-1}}+c_{r_{i}}
$$

leads to a solution of the problem. If not, $x=a_{r_{i+1}}$ with $1 \leqq r_{i+1} \leqq n-2$ and we proceed to the $(i+1)$ th stage by replacing $b_{r_{i+1}}$ and $c_{r_{i+1}}$ by $b_{r_{i}}$ and $c_{r_{i-1}}$. Hence either (1) we reach a solution of the problem or (2) the process continues indefinitely. We shall show that the second alternative cannot arise. In the second alternative since $a_{r_{1}}, a_{r_{2}}, \ldots$ are drawn from the finite set $a_{1}, \cdots, a_{n-2}$, there will be indices $i$ and $j \geqq i$ such that $a_{r_{1}}, \cdots, a_{r_{i}}, \cdots, a_{r_{j}}$ are all distinct, but $a_{r_{j+1}}=a_{r_{i}}$. Then at the $j$ th stage we have

$$
\begin{array}{ll}
a_{1} \cdots a_{r_{i}} \cdots a_{r_{j}} \cdots a_{n-2} a_{n-1} a_{n} & \\
b_{1} \cdots b_{r_{i-1}} \cdots b_{r_{j-1}} \cdots b_{n-2} & b_{r_{j}} \quad b_{n} \\
c_{1} \cdots c_{r_{i-2}} \cdots c_{r_{j-2}} \cdots c_{n-2} & c_{r_{j-1}} c_{r_{j}}
\end{array}
$$

and the solution of $x+b_{r_{j}}=c_{r_{j-1}}$ is $x=a_{r_{i}}$. At the $(j+1)$ th stage the $b$ 's and $c$ 's left over are

$$
\begin{aligned}
& b_{r_{i-1}} b_{n} \\
& c_{r_{j}} \quad c_{r_{i-2}}
\end{aligned}
$$

whence

$$
a_{n-1}+a_{n}+b_{r_{i-1}}+b_{n}=c_{r_{j}}+c_{r_{i-2}} \text {. }
$$

But at the $(i-1)$ th stage we had (from (2.7) or (2.3) if $i=1$ )

$$
a_{n-1}+a_{n}+b_{r_{i-1}}+b_{n}=c_{r_{i-2}}+c_{r_{i-1}} \text {. }
$$

Comparing (2.10) and (2.11) we find that

$$
c_{r_{j}}=c_{r_{i-1}} .
$$

But this is a contradiction since $j>i-1$ and $c_{r_{j}}$ and $c_{r_{i-1}}$ are distinct elements in (2.8). Thus the second alternative does not arise and we 
find a solution to the problem in not more than $n-2$ steps.

3. Application to Latin squares. Consider a Latin square which is the Cayley table for an abelian group of order $n$

$$
\begin{aligned}
& a_{11}, a_{12}, \cdots, a_{1 n} \\
& a_{21}, a_{22}, \cdots, a_{2 n} \\
& \cdot \cdot \cdot \cdot \cdot \cdot \cdot \\
& a_{n 1}, a_{n 2}, \cdots, a_{n n} .
\end{aligned}
$$

Here if $a_{1}=0, a_{2}, \cdots, a_{n}$ are the elements of $A$, then in the table above $a_{i j}=a_{i}+a_{j}$. If

$$
\left(\begin{array}{l}
a_{1}, \cdots, a_{n} \\
c_{1}, \cdots, c_{n}
\end{array}\right)
$$

is a permutation of the elements of $A$, then $c_{r}$ is below $a_{r}$ in the $k$ th row if $c_{r}-a_{r}=b_{r}=a_{k}$. We say that $c_{1}, c_{2}, \cdots, c_{r}, \cdots, c_{n}$ agrees with the $k$ th row in position $r$. Thus the theorem asserts that there exists a permutation agreeing with the $i$ th row $k_{i}$ times if and only if

$$
k_{1}+k_{2}+\cdots+k_{n}=n,
$$

and

$$
k_{1} a_{1}+k_{2} a_{2}+\cdots+k_{n} a_{n}=0,
$$

where (3.2.1) is a count of the $k$ 's and (3.2.2) is an equation in $A$. The sum of all the elements of an abelian group $A$ is known to be 0 unless $A$ contains a unique element of order 2 , in which case the sum is this unique element. In the special case in which $k_{1}=k_{2}=\ldots$ $=k_{n}=1$ we say that $c_{1}, \cdots, c_{n}$ is a transversal of the Latin square. Here (3.2.2) does not hold if $A$ contains a unique element of order 2 and there is no transversal. But if $A$ does not contain a unique element of order 2, then (3.2.2) does hold and there is a transversal of the Latin square. This special case of the theorem above was proved by Lowell Paige in his doctoral dissertation at the University of Wisconsin.

OHio State University 